\title{
Non-random gene flow: an underappreciated force in evolution and ecology
}

\author{
Pim Edelaar ${ }^{1,2}$ and Daniel I. Bolnick ${ }^{3}$ \\ ${ }^{1}$ Department of Conservation Biology, Estación Bioló gica de Doñana, Consejo Superior de Investigaciones Científicas (CSIC), \\ E-41092, Sevilla, Spain \\ ${ }^{2}$ Department of Molecular Biology and Biochemistry Engineering, University Pablo de Olavide, E-41013, Sevilla, Spain \\ ${ }^{3}$ Howard Hughes Medical Institute, and Section of Integrative Biology, University of Texas at Austin, Austin, TX 78712, USA
}

Dispersal is an important life-history trait involved in species persistence, evolution, and diversification, yet is one of the least understood concepts in ecology and evolutionary biology. There is a growing realization that dispersal might not involve the random sample of genotypes as is typically assumed, but instead can be enriched for certain genotypes. Here, we review and compare various sources of such non-random gene flow, and summarize its effects on local adaptation and resource use, metapopulation dynamics, adaptation to climate change, biological invasion, and speciation. Given the possible ubiquity and impacts of non-random gene flow, there is an urgent need for the fields of evolution and ecology to test for non-random gene flow and to more fully incorporate its effects into theory.

\section{Rethinking the homogenizing effect of gene flow}

Theory suggests that evolutionary change depends on the action of a limited number of fundamental biological processes that: (i) introduce novel genetic variation into populations (mutation, recombination, and gene flow; see Glossary), or (ii) change the relative frequencies of existing genotypes (natural, sexual and social selection, genetic drift, assortative mating, and various forms of intragenomic conflict). Of these processes, only natural selection is widely believed to drive adaptive evolution [1,2], in the sense of deterministically increasing the mean fitness of a population within its native habitat.

The remaining evolutionary processes are typically viewed as sources of genetic variation for selection to act upon [3-5], or as forces that constrain the adaptive effects of natural selection [6-9]. For example, gene flow is widely assumed to counteract the effect of natural selection [8] by introducing maladaptive foreign alleles into a locally adapted population [10]. Most evolutionary models assume that dispersal is random with respect to genotype $[3,8,11]$. That is, in each generation, some fraction of individuals $\mathrm{m}_{\mathrm{ij}}$ disperse from population i to population $\mathrm{j}$, and these dispersers carry the same allele frequency as the donor population i (or for small populations, a random sample of donor alleles). Such random gene flow tends to make spatially separate populations more genetically similar (e.g., lower indices of genetic differentiation such as $\mathrm{F}_{\mathrm{ST}}$ ) and, consequently, tends to reduce mean fitness if those populations are subject to divergent natural selection ('migration load').

However, it is becoming increasingly clear that gene flow can have a far more complex role in evolution than generally believed [12-16]. Thus, it is time for a substantial rethinking of the emphasis of evolutionary theory on the

\section{Glossary}

Adaptive or matching habitat choice: when individuals use some measure of local performance to establish a preference for settlement in one of several habitats in order to increase their expected fitness. When different genotypes choose different optimal habitats, such habitat choice causes individuals to disperse in a manner that leads to a match between phenotype and environment.

Condition-dependent dispersal: dispersal that depends on the environmental conditions, often because these affect the costs and benefits of available dispersal decisions. Confusingly, also used to mean dispersal that depends on the physical condition of the disperser (e.g., level of energy reserves). Dispersal: the movement and incorporation of individuals between populations. Dispersal can be broken down into a departure, transience, and settlement stage, each of which can be active (at least under partial behavioral control of the individual) or passive. Dispersal does not necessarily imply gene flow, unless dispersers change allele frequencies in their destination population.

Gene flow: the movement and incorporation of alleles between populations. Gene leakage: a change in allele frequency in a source population due to genotypically biased emigration.

Migration: (i) an evolutionary term referring to the movement of alleles from one population to another (i.e., gene flow); or (ii) repeated and often cyclic movement of individuals between the same geographical areas (e.g., between wintering and breeding ranges). This ecological meaning of migration is not considered further in this opinion article.

Migration load: the reduction in mean fitness of a population due to immigration of locally maladapted alleles.

Migration-selection balance: an equilibrium level of between-population genetic divergence, reflecting the divergent effect of natural selection and the homogenizing effect of random gene flow.

Movement: a change in the physical location of an individual through time. When the individual moves to, and is incorporated into, another population, we call it dispersal.

Non-random gene flow versus random gene flow: gene flow is random for a given trait (e.g., morphology, physiology or behavior, type of current habitat, or genotype) if all dispersal characteristics of individuals (i.e., dispersal probability, distance, or destination) are uncorrelated with the genetic variation in this trait within a population. If such correlation does exist, then gene flow is non-random with respect to the correlated trait(s). Note that random gene flow does not necessarily imply Brownian motion through space: individuals might still prefer certain habitats or move in a directed manner through space, but gene flow remains random as long as all genotypes are equally likely to disperse in the same manner. We also use 'non-random dispersal' and 'random dispersal' as analogs of non-random and random gene flow, respectively, for the case where dispersal-influencing traits are not heritable, or heritability is unknown. 
constraining effects of gene flow. Here, we review and synthesize evidence that gene flow can be biased with respect to genotype; this bias can lead to qualitatively different effects on adaptation and population differentiation. We identify numerous distinct sources of genetic bias in gene flow, discuss the implications of non-random gene flow for evolution, ecology, and conservation biology, and call for increased empirical and theoretical attention to be paid to this generally overlooked phenomenon.

\section{Sources of bias in gene flow}

Below, we present several sources that can lead to genotype-dependent dispersal. This happens when genetic variation leads to intraspecific variation in dispersal behavior, generating gene flow that is non-random with respect to individual variation. Note that this implies a cause-effect directionality in which pre-existing individual variation causes variation in dispersal. Thus, individual variation is a prerequisite. This differs from another form of individual variation in dispersal behavior increasingly acknowledged in ecological studies of dispersal (e.g., [14,17-19], wherein variation in environmental conditions affects the dispersal decisions of individuals (conditiondependent dispersal), which then adapt their phenotypes in response to these decisions. Individual variation is the output. The two forms of dispersal variation can coincide when there is genetic variation in the reaction norms connecting environmental conditions to dispersal behavior, such that some genotypes are more predisposed than others to undergo condition-dependent dispersal.

The sources of bias listed below share two key elements: (i) pre-existing among-individual variation in certain traits within the population; and (ii) a causal link between this individual variation and some aspect of dispersal. The list of sources might not be exhaustive, but should cover most sources of non-random gene flow, which can generally be characterized by variation in habitat preferences, dispersal capacity, and/or performance-dependent dispersal. These sources are not mutually exclusive (Figure 1) and might have additive or interactive effects, but presenting them separately helps to illustrate the diverse causes and potential generality of non-random gene flow.

\section{Source 1: performance variation}

If individuals can sense some aspect of their performance (e.g., stress or hunger) that is correlated with fitness, they could potentially use this information to make informed dispersal decisions [14]. Individuals should be more likely to disperse if they: (i) have low local performance; or (ii) detect the possibility of higher performance at a foreign site. Local performance might be judged relative to some threshold (e.g., perceived vulnerability to local predators [20]), or might be relative to other conspecific individuals within the same habitat (e.g., ability to defend a breeding territory [21]). Alternatively, individuals might sample multiple habitat sites to evaluate their performance, and select the site expected to confer highest fitness [22]. Perhaps the best example of performance-dependent dispersal is the Wrangler grasshopper Circotettix rabula: predation risk is reduced when the body color of a grasshopper matches its background [23], whereas contrasting individuals (e.g., experimentally painted) move to another habitat to restore crypsis [24].

\section{Source 2: personality variation}

Many studies document consistent behavioral differences among individuals (sometimes termed behavioral types, personalities, or behavioral syndromes [25]), which can be correlated with dispersal (reviewed in [26]). For example, great tits Parus major exhibiting more exploration,

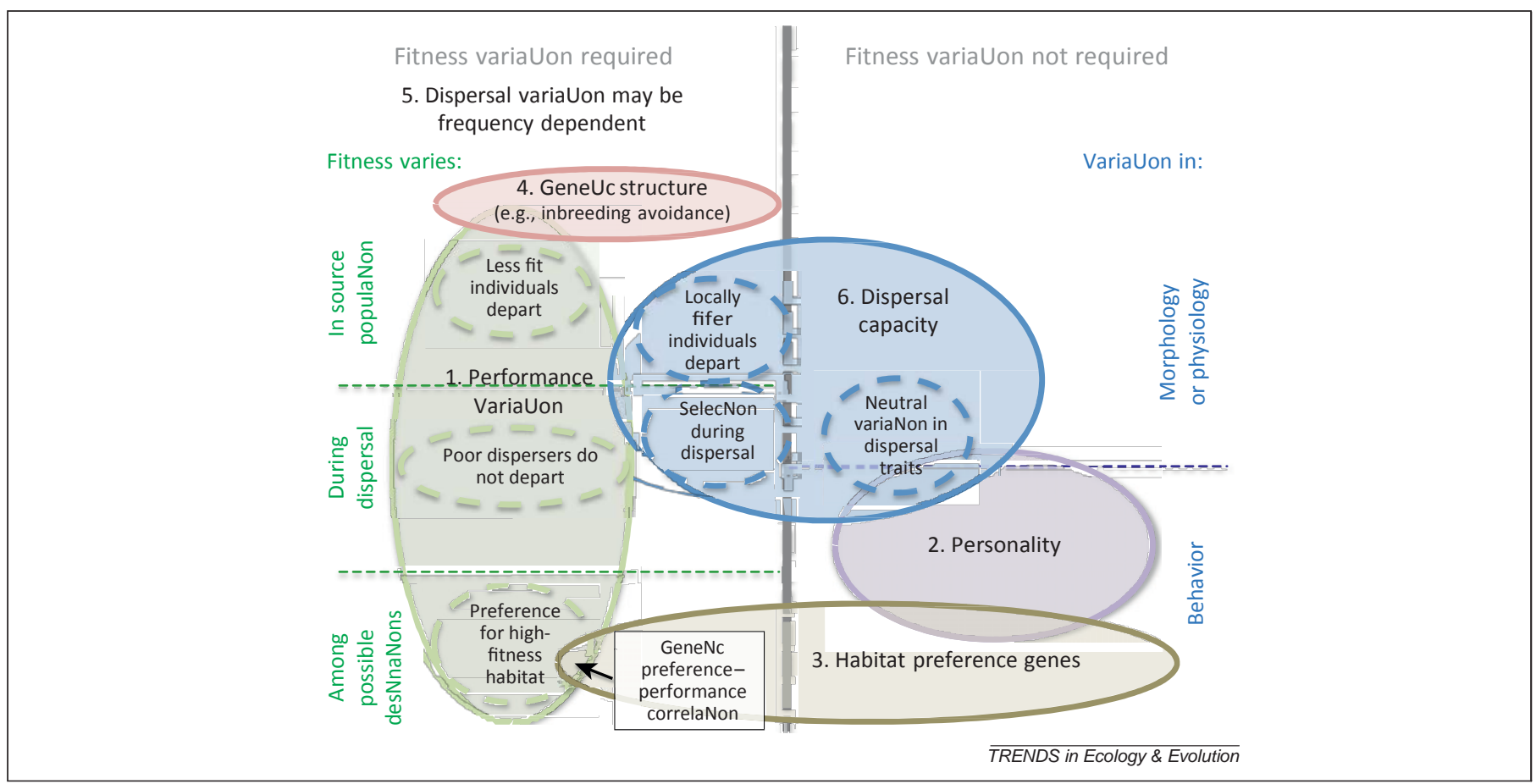

Figure 1. Schematic overview of sources underlying non-random gene flow. Indicated are their relations with several criteria, and conceptual similarities between certain sources (indicated by partial overlap). 
aggression, and boldness dispersed further in the wild [27]. In the fruit fly Drosophila melanogaster, individuals showed heritable variation in whether they settled on food patches that had no, few, or many conspecifics, resulting in a correlation between genetically determined sociality and social environment [28]. Behavioral sex differences in dispersal are an especially common special case of personality-dependent dispersal, and can generate important differences in the geographic structure of male- and female-specific genes [21,29].

\section{Source 3: habitat preference genes}

The choice to settle in one habitat over another might have a direct genetic basis that can differ among individuals. Habitat preferences can be controlled by genes affecting the physiological capacity of an organism to detect habitat cues, or its neurological ability to orient towards such cues. For instance, many phytophagous insects exhibit preferences for (or aversions to) the scent of particular host plants (reviewed in [30,31]). Such preferences sometimes coincide with higher performance in the preferred habitat [32] (see also Figure 1) but, in other cases, preference and performance are decoupled (e.g., [33-35]). Consequently, we separate the idea of genetic variation in habitat preference, from preference arising pleiotropically from genetic variation in performance in a habitat (source 1, above).

\section{Source 4: genetic structure}

Dispersal often aims to reduce kin competition and/or inbreeding, so individuals with higher mean relatedness coefficients with interacting individuals might be more likely to disperse. Conversely, many species exhibit a tendency to avoid outbreeding, selecting against dispersal. Therefore, genotypes within a given population might differ in their dispersal behavior, depending on their relatedness to others. For example, the invasive fire ant Solenopsis invicta displays genetic differentiation in sympatry between the single-queen and multiple-queen social form, because dispersing females have great difficulty establishing and entering colonies of the alternative form [36].

\section{Source 5: frequency dependence}

The fitness of an individual might depend on interactions with other individuals in a way that leads to frequencydependent selection. Individuals might benefit from associating with phenotypically dissimilar conspecifics (e.g., if predators form search images for locally common prey types), or with similar conspecifics (e.g., in collaborating or aposematic species). Therefore, individuals might select a habitat based on the local phenotype distribution, similar to performance-dependent dispersal except that the relevant habitat variable is the composition of conspecifics. Frequency dependence can also bias gene flow via more passive processes. For example, in heterostylous plants, matings among individuals of the same morph are usually incompatible. Hence, the pollen of a locally rare morph is more likely to fertilize individuals within the same population (i.e., low dispersal), whereas pollen of locally common morphs is more likely to fertilize individuals in other populations [37].

\section{Source 6: dispersal capacity variation}

When individuals vary in biomechanical or physiological dispersal capacities, then they can correspondingly vary in dispersal probability or distance. For example, Glanville fritillary butterflies Melitaea cinxia that carry a certain allelic variant of the Pgi locus have a greater flying capacity [38]. Note that dispersal capacity variation can be selectively neutral, but can also lead to selective mortality during transience. As an example of the latter, deep-bodied sockeye salmon Oncorhynchus nerka individuals are more likely to die (from predation by bears or from stranding) when dispersing from a lake into a shallow stream, contributing to genetic divergence between small-bodied upstream and more variable lake-breeding populations [39]. The effects of selection during dispersal can also be delayed, arising via reduced fecundity or longevity of poor dispersers after arrival at a new site (e.g., in the tropical butterfly Bicyclus anynana [40]). Capacity-dependent dispersal can co-vary with local performance (see Source 1: performance variation), for instance if locally betteradapted individuals have higher energy reserves for dispersal (see 'condition-dependent dispersal' in Glossary). Ironically, this could contribute to local maladaptation via 'gene leakage' of locally adapted genotypes.

To summarize, there is a host of sources of biased gene flow between populations in different environments or locations (Figure 1). Importantly, biases in gene flow can result in changes in population genetic composition (i.e., evolution), but this can happen for reasons that are unrelated to fitness. For example, during range expansion or biological invasion, individuals with greater passive dispersal capacities are more likely to be found in newly establishing edge populations, not because they have higher fitness there, but just because they have greater dispersal distances. Interestingly, this will mean that the speed of range expansion evolves to increase as an unselected byproduct of dispersal capacity variation [13]. Of course, if these more dispersive individuals do enjoy a higher fitness in newly establishing populations (e.g., due to reduced competition), then natural selection will further accelerate the evolution of greater dispersal capacities [13]. The relative effects of these two components in an empirical range expansion have yet to be determined.

\section{Non-random gene flow as a core concept}

The within-population evolutionary processes can be categorized based on whether they typically act randomly on genotypes (e.g., mutation, recombination, or genetic drift) or act non-randomly on genotypes (selection, assortative mating, or processes of intragenomic conflict). Whether a process is random or non-random with respect to genotype has a huge importance: it determines whether the dynamics of a genotype are stochastic or deterministic, and whether it can lead to adaptation. Given this impact, it is imperative to also consider whether the main betweenpopulation evolutionary process (i.e., gene flow) is random or non-random with respect to genotype, and the consequences of this distinction. Random gene flow has been the standard assumption of evolutionary genetic models [3,8], and this might be appropriate for most loci within a genome. However, few evolutionary models consider 
whether gene flow is random or non-random with respect to functional genotypes, and mostly these directly model the evolution of the dispersal traits themselves (e.g., [41]). The non-randomness of gene flow was of substantial interest during the 1960s and 1970s [42-45], then was largely dropped and has only recently gained new attention [1214,46-49].

Therefore, we propose that evolutionary theory needs to incorporate explicitly the idea of 'non-random gene flow' as a core concept. We define non-random gene flow as any aspect of dispersal (e.g., dispersal probability, distance or direction, or location of settlement) of individuals or gametes that is deterministically biased with respect to the genetic variation in the population from which the individuals or gametes originated. We distinguish this from non-random dispersal, which also includes dispersal that is biased with respect to non-genetic aspects of phenotypic variation, for instance arising from habitat imprinting [50,51], habitat training [52,53], parental manipulation [54,55], or other sources of phenotypic plasticity. Sources of genetic and non-genetic variation could act in concert to bias dispersal, for instance if there is heritable variation in the reaction norms for phenotypic plasticity underlying dispersal variation. As an aside, the term 'non-random dispersal' (including condition-dependent dispersal) is also sometimes used to describe dispersal that is biased with respect to environmental variation (e.g., population density, patch size, or habitat suitability), but here we are concerned with bias in dispersal with respect to individual variation.

Non-random gene flow provides an encompassing term for the population genetic effects of all previously rather disconnected and idiosyncratic sources of bias in dispersal outlined above. By introducing this term as the overarching evolutionary process of dispersal that is not independent of genotype, we fill in a hitherto neglected component of evolutionary theory that deserves more explicit consideration, both in population genetics and quantitative genetics theory, as well as empirically.

Consequences of non-random gene flow and dispersal The consequences of non-random gene flow and dispersal can be substantially different from the typical consequences of random gene flow and dispersal. Below, we highlight these qualitative differences by contrasting various evolutionary and ecological effects of random and nonrandom gene flow and dispersal.

\section{Migration-selection balance and local adaptation}

Classic evolutionary theory suggests that gene flow tends to constrain genetic differentiation among populations subject to divergent selection [11,56], and to reduce mean fitness [57] (except if immigrants happen to introduce new but locally adaptive alleles). These detrimental effects of gene flow have been extensively analyzed theoretically and documented empirically [8]. However, non-random gene flow might lead to individuals departing areas of low fitness and settling in areas of high fitness, in which case gene flow might actually increase mean fitness and promote among-habitat divergence [12]. Hence, gene flow might in fact account for a large portion of the process of adaptive divergence (Box 1). Consequently, the widely accepted maladaptive and homogenizing effect of gene flow can be completely reversed when dispersal is somehow related to fitness after settlement.

\section{Species ranges and ecological niches}

Random gene flow from well-established, adapted populations into ecologically different, novel habitats might impose a sufficient migration load to cause low fitness or extinction of geographically or ecologically peripheral populations. Therefore, maladaptive gene flow has been invoked as an explanation for the existence of limits to the geographic ranges and ecological niches of species [58,59]. Non-random gene flow might instead help explain patterns of rapid range expansion, when the range edge of a species becomes enriched for highly dispersive phenotypes that are also better able to occupy new territory [13,60]. For example, the western bluebird (Sialia mexicana) expanded its range because biased dispersal of highly aggressive males to the invasion front allowed them to displace less aggressive mountain bluebirds (Sialia currucoides) [61]. Conversely, habitat imprinting, habitat learning, or genetic habitat preferences from within the established range could place additional constraints on the geographical or ecological range of a species: if individuals are behaviorally disinclined to disperse into adjoining habitat, geographical, or ecological ranges will be slower to change.

\section{Genomic analyses of divergent selection}

An increasingly common method of finding the genetic basis of adaptation is to sample populations across a range of habitats, and then scan the genome for regions of exceptional between-population divergence. Such divergent chromosomal regions are typically assumed to be subject to spatially divergent selection [62-64]. An alternative interpretation is that genetic markers exhibiting exceptionally high (or low) divergence are linked to genes causing non-random gene flow. For example, alleles that encode habitat preference or dispersal capacity can show between-habitat frequency differences even if they are not adaptive, simply because genotypes sort non-randomly across space [13]. If non-random gene flow is an appreciable evolutionary force, then population genomics must grapple with how to distinguish whether highly divergent regions in the genome represent the effects of natural selection or of non-random gene flow.

\section{Speciation}

To the extent that phenotypic or genetic divergence between populations drives reproductive isolation and, thus, speciation, homogenizing gene flow represents a constraint on the rate of speciation. This constraint is why allopatric speciation is typically considered far easier to achieve than is parapatric speciation, and why sympatric speciation (where gene flow is initially unlimited) is often considered quite difficult [65]. Conversely, non-random gene flow might substantially increase the feasibility of sympatric or parapatric speciation $[43,66]$. For instance, habitat preferences by lake and stream three-spined stickleback drastically reduced the rate of dispersal between these adjoining habitat types, thereby promoting assortative 
To what extent populations diverge genetically over time depends on several processes. Typically, it is thought that natural selection is the only process that can drive adaptive population divergence, and that all other processes act towards the erosion of adaptive divergence. Here, we argue that non-random gene flow can also promote adaptive population divergence, and could even create adaptive divergence without any contribution by natural selection (defined here as genotype-dependent variance in fecundity or survival). For this, we contrast three scenarios involving natural selection, random gene flow, and non-random gene flow, and explore their dynamic consequences for adaptive population differentiation over time (Figure I).

If divergent natural selection is absent, then random gene flow will homogenize populations (Figure I, black lines). Depending on their initial level of divergence, this will go slower or faster. However, if populations are exposed to some divergent natural selection, then selection and gene flow will result in a dynamic equilibrium level of divergence (i.e., migration-selection balance). If divergence is initially below this equilibrium, then natural selection will increase the level of divergence (Figure I, lower blue line), whereas if divergence is initially above this equilibrium, then gene flow will decrease the level of divergence (Figure I, upper blue line).

Finally, we add a certain amount of non-random gene flow in the form of performance-dependent dispersal to the system, such that individuals are more likely to move towards the habitat in which their fitness is highest. This will increase the equilibrium level of divergence. Initially, little-diverged populations will increase their divergence, due to both divergent natural selection and to nonrandom gene flow (Figure I, lower unbroken red line). However, when the fitness-dependent habitat selection is not $100 \%$ correct (as in Figure I), then gene flow still has a random component to it. If the populations were initially highly diverged, then divergence can still decrease despite gene flow being non-random (Figure I, upper unbroken red line). Hence, whether and at what rate a process increases or decreases divergence depends on its strength and on the initial level of divergence relative to the equilibrium level of divergence.

mating and allowing genetic divergence to build up between populations just tens of meters apart [15]. Habitat imprinting and host plant preferences are also widely invoked to explain speciation in phytophagous insects $[31,67]$. Thus, the degree to which gene flow and dispersal are random might substantially affect the speed and geographic context of speciation.

\section{Conservation biology and metapopulation dynamics}

For conservation purposes, is it best to protect large contiguous blocks of habitat, or smaller more heterogeneous habitats, or disjunct islands of divergent habitats? Nonrandom dispersal of native taxa is not typically considered during such decisions, and little is known of how spatial habitat arrangements interact with various types of nonrandom dispersal. However, it is conceivable that such interactions are important for species persistence. For example if dispersal depends on relatedness with neighbors (source 4, above), then threatened species, which are subjected to increasing inbreeding, might be more dispersive in the future than they currently are. In metapopulations, locally extinct or more isolated populations will receive immigrants that are biased towards greater dispersal [38], which can have demographic effects (e.g., reduced fecundity) and evolutionary effects (e.g., genetic increase of dispersal distance). Conversely, reduced habitat quality and connectivity could select against dispersal,
An unrealistic but insightful example of this increased adaptation is when fitness-dependent dispersal is perfect and unconstrained. This implies that all individuals can perform a complete assessment of their relative fitness in alternate habitats, are free to move to the habitat were they are expected to do best, and can do so instantaneously and without costs. Under such conditions, population divergence will be maximal because no single individual will occur in the non-optimal habitat (Figure I, broken red lines). This also means that there will have been no time for selective differential mortality or differential reproduction to occur. Thus, in this extreme example, adaptive divergence has occurred even in the absence of divergent natural selection. Therefore, non-random gene flow is a distinct evolutionary force capable of driving adaptive evolution, in addition to natural selection.

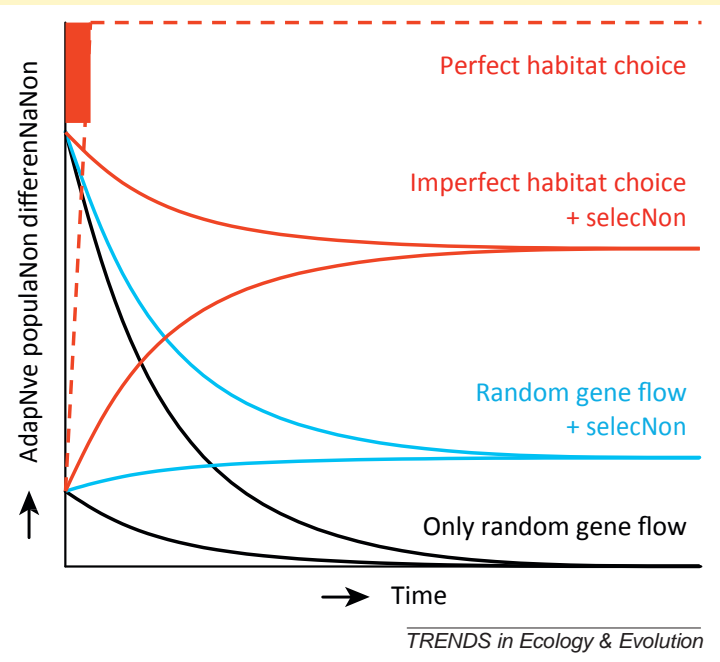

Figure I. Random gene flow reduces population differentiation, but non-random gene flow can increase it.

resulting in increased inbreeding within more isolated populations [19]. Habitat imprinting or training might prove important in re-establishment of endangered species from captive stock, to ensure released individuals remain within protected areas [16].

\section{Concluding remarks}

The study of non-random gene flow and dispersal is still in its infancy. There are enough empirical studies to make it clear that non-random dispersal happens, and arises from a wide variety of sources. However, we know little of the cognitive, physiological, or genetic basis of non-random dispersal in most species [68]. Neither do we know how widespread non-random dispersal is in nature, its typical effect size, or its evolutionary effects, although there is ample reason to think that they are important. Clarifying these issues will require a combination of laboratory and fieldwork on dispersal and dispersive decision making, population genomic analyses, and new theoretical models.

In particular, there is a substantial need to rework population genetics theory to create a general mathematical framework that can accommodate the qualitatively distinct effects of non-random gene flow. There is also a need for more models that investigate under what conditions non-random dispersal will or will not evolve, which specific sources of non-random gene flow are expected to evolve, and how these interact and feedback with natural 


\section{Box 2. Testing for non-random gene flow or dispersal}

There are several complementary approaches to test for nonrandom gene flow or dispersal (see main text 'Sources of bias in gene flow' for published examples). A descriptive-comparative test of non-random dispersal is to compare the phenotype distributions of individuals belonging to different classes, such as: (i) residents versus dispersers; (ii) successful and unsuccessful dispersers; (iii) individuals that disperse short or long distances; (iv) individuals that disperse in different directions; or (v) individuals that settle in alternate habitats from the same initial source. One must be careful to control for site of origin to avoid spurious results arising from genetic spatial structure.

One should not restrict oneself to only comparing these endpoints of the dispersal process: there is much information to be gained by paying closer attention to what individuals do, for example, which individuals explored but did not depart, or which sites were visited during transience but not selected for settlement, or in which habitats did selective dispersal mortality occur. Thanks to modern development of all sorts of tracking devices and techniques with suitable spatial and temporal resolutions, such spatiotemporal tracking of dispersing individuals from whales to zooplankton [69] has become easier, and we encourage its incorporation in studies of dispersal.

Whether gene flow is non-random can be determined by several methods, either in the wild or under controlled laboratory conditions. Classical quantitative or population genetic tools can be used to determine the heritability of variation in dispersal, for example, by parent-offspring regression, animal modeling, or measuring the response to selection. Alternatively, or to obtain a more detailed insight, one could use genetic mapping or candidate gene approaches to identify the actual gene(s) influencing variation in dispersal.

Experimental approaches to test for non-random gene flow or dispersal can be very insightful, and we discuss three techniques (which can be combined). (i) One could experimentally manipulate the environmental characteristics that are believed to make dispersal non-random, such as predation risk or frequency of related individuals, and test for non-random dispersal as a response. (ii) A mirror image of the first approach is to manipulate the individuals (e.g., performance, personality, inbreeding coefficient, or dispersal capacity) via selective breeding, induced phenotypic plasticity, or direct phenotypic manipulations, and test for nonrandom dispersal as a response. (iii) One could artificially displace individuals, and follow their subsequent dispersal to test whether staying, returning, or moving to yet another habitat is related to individual traits.

selection and phenotypic plasticity in driving adaptive population divergence.

There is also a desperate need for more solid case studies. In Box 2, we provide some suggestions on how to test empirically for non-random dispersal and gene flow. A major challenge can be the identification of the relevant genetic variation to study. Most genes in the genome will probably exhibit random gene flow so non-random gene flow, similar to natural selection [62,63], might lead to chromosomal islands of high divergence among populations. Therefore, unless a researcher has strong candidate genes, studies of non-random dispersal might benefit from use of whole genomes or large panels of single nucleotide polymorphisms (SNPs) to identify these regions successfully. Alternatively, studies focusing on heritable quantitative traits might be more successful, especially if researchers have clear functional reasons to suspect that particular traits might cause biased dispersal.

Evolutionary and ecological research will benefit from explicitly testing for the non-randomness of dispersal and gene flow, and evolutionary theory will be greatly enriched by its incorporation. Therefore, we propose to view gene flow as potentially ranging from random to fully deterministic, and to treat random gene flow as a statistical null hypothesis that should be tested with data as much as possible, instead of assumed to be true.

\section{Acknowledgments}

We thank Yuexin Jiang, Maria Del Mar Delgado, Roger Shaw, and three anonymous reviewers for comments on earlier versions of this paper. This article was supported by the Ministry of Science of Spain (JAE-Doc and Ramon y Cajal contracts), the David and Lucille Packard Foundation, and the Howard Hughes Medical Institute.

\section{References}

1 Rose, M.R. and Lauder, G.V., eds (1996) Adaptation, Academic Press

2 Endler, J.A. (ed.) (1986) Natural Selection in the Wild, Princeton University Press

3 Rä sä nen, K. and Hendry, A.P. (2008) Disentangling interactions between adaptive divergence and gene flow when ecology drives diversification. Ecol. Lett. 11, 624-626

4 Kopp, M. and Hermisson, J. (2009) The genetic basis of phenotypic adaptation I: fixation of beneficial mutations in the moving optimum model. Genetics 182, 233-249

5 Orr, H.A. (1998) The population genetics of adaptation: the distribution of factors fixed during adaptive evolution. Evolution 52, 935-949

6 Burger, R. and Lande, R. (1994) On the distribution of the mean and variance of a quantitative trait under mutation-selection-drift balance. Genetics 138, 901-912

7 Yeaman, S. and Whitlock, M.C. (2011) The genetic architecture of adaptation under migration-selection balance. Evolution 65, 18971911

8 Lenormand, T. (2002) Gene flow and the limits to natural selection. Trends Ecol. Evol. 17, 183-189

9 Crespi, B.J. (2000) The evolution of maladaptation. Heredity 84, 623629

10 Bolnick, D.I. and Nosil, P. (2007) Natural selection in populations subject to a migration load. Evolution 61, 2229-2243

11 Slatkin, M. (1985) Gene flow in natural populations. Annu. Rev. Ecol. Syst. 16, 393-430

12 Edelaar, P. et al. (2008) Matching habitat choice causes directed gene flow: a neglected dimension in evolution and ecology. Evolution 62, 2462-2472

13 Shine, R. et al. (2011) An evolutionary process that assembles phenotypes through space rather than evolutionary time. Proc. Natl. Acad. Sci. U.S.A. 108, 5708-5711

14 Clobert, J. et al. (2009) Informed dispersal, heterogeneity in animal dispersal syndromes and the dynamics of spatially structured populations. Ecol. Lett. 12, 197-209

15 Bolnick, D.I. et al. (2009) Phenotype-dependent native habitat preference facilitates divergence between parapatric lake and stream stickleback. Evolution 63, 2004-2016

16 Stamps, J.A. and Swaisgood, R.R. (2007) Someplace like home: experience, habitat selection and conservation biology. Appl. Anim. Behav. Sci. 102, 392-409

17 Bowler, D.E. and Benton, T.G. (2005) Causes and consequences of animal dispersal strategies: relating individual behaviour to spatial dynamics. Biol. Rev. 80, 205-225

18 Ronce, O. (2007) How does it feel to be like a rolling stone? Ten questions about dispersal evolution. Annu. Rev. Ecol. Evol. Syst. 38, 231-253

19 Clobert, J. et al. (2004) Causes, mechanisms, and consequences of dispersal. In Ecology, Genetics and the Evolution of Metapopulations (Hanski, I. and Gaggiotti, O.E., eds), pp. 307-335, Academic Press

20 Skov, C. et al. (2010) Sizing up your enemy: individual predation vulnerability predicts migratory probability. Proc. R. Soc. B: Biol. Sci. $278,1414-1418$

21 Cano, J.M. et al. (2008) Genetic evidence for male-biased dispersal in three-spined stickleback (Gasterosteus aculeatus). Mol. Ecol. 17, 32343242

22 Ahnesjö , J. and Forsman, A. (2006) Differential habitat selection by pigmy grasshopper color morphs; interactive effects of temperature and predator avoidance. Evol. Ecol. 20, 235-257 
23 Cox, G.W. and Cox, D.G. (1974) Substrate color matching in the grasshopper, Circotettix rabula (Orthoptera: Acrididae). Great Basin Nat. 34, 60-70

24 Gillis, J.E. (1982) Substrate colour-matching cues in the cryptic grasshopper Circotettix rabula rabula (Rehn \& Hebard). Anim. Behav. 30, 113-116

25 Sih, A. et al. (2004) Behavioral syndromes: an ecological and evolutionary overview. Trends Ecol. Evol. 19, 372-378

26 Cote, J. et al. (2010) Personality-dependent dispersal: characterization, ontogeny and consequences for spatially structured populations. Philos. Trans. R. Soc. B 365, 4065-4076

27 Dingemanse, N.J. et al. (2003) Natal dispersal and personalities in great tits (Parus major). Proc. R. Soc. B: Biol. Sci. 270, 741-747

28 Saltz, J.B. (2011) Natural genetic variation in social environment choice: context-dependent gene-environment correlation in Drosophila melanogaster. Evolution 65, 2325-2334

29 Bolnick, D.A. et al. (2006) Asymmetric male and female genetic histories among Native Americans from eastern North America. Mol. Biol. Evol. 23, 2161-2174

30 Jaenike, J. and Holt, R.D. (1991) Genetic variation for habitat preference: evidence and explanations. Am. Nat. 137, S67-S90

31 Berlocher, S.H. and Feder, J.L. (2002) Sympatric speciation in phytophagous insects: moving beyond controversy? Annu. Rev. Entomol. 47, 773-815

32 Hawthorne, D.J. and Via, S. (2001) Genetic linkage of ecological specialization and reproductive isolation in pea aphids. Nature 412, 904-907

33 Gratton, C. and Welter, S.C. (1998) Oviposition preference and larval performance of Liriomyza helianthi (Diptera: Agromyzidae) on normal and novel host plants. Environ. Entomol. 27, 926-935

34 Agashe, D. (2009) The stabilizing effect of intraspecific genetic variation on population dynamics in novel and ancestral habitats Am. Nat. 174, 255-267

35 Agashe, D. and Bolnick, D.I. (2010) Intraspecific genetic variation and competition interact to influence niche expansion. Proc. R. Soc. B: Biol. Sci. 277, 2915-2924

36 Shoemaker, D.D. and Ross, K.G. (1996) Effects of social organization on gene flow in the fire ant Solenopsis invicta. Nature 383, 613-616

37 Barrett, S.C.H. (1990) The evolution and adaptive significance of heterostyly. Trends Ecol. Evol. 5, 144-148

38 Hanski, I. (2011) Eco-evolutionary spatial dynamics in the glanville fritillary butterfly. Proc. Natl. Acad. Sci. U.S.A. 108, 14397-14404

39 Lin, J. et al. (2008) Fine-scale differentiation between sockeye salmon ecotypes and the effect of phenotype on straying. Heredity 101, 341-350

40 Saastamoinen, $M$. et al. (2010) Predictive adaptive responses: condition-dependent impact of adult nutrition and flight in the tropical butterfly Bicyclus anynana. Am. Nat. 176, 686-698

41 Travis, J.M.J. et al. (2010) Towards a mechanistic understanding of dispersal evolution in plants: conservation implications. Divers. Distrib. 16, 690-702

42 Fretwell, S.D. and Lucas, H.L. (1969) On territorial behavior and other factors influencing habitat distribution in birds. I. Theoretica development. Acta Biotheor. 19, 16-36

43 Maynard Smith, J. (1966) Sympatric speciation. Am. Nat. 100, 637-650

44 Jaenike, J. (1978) Host selection by mycophagous Drosophila. Ecology 59, 1286-1288

45 Bush, G.L. (1975) Modes of animal speciation. Annu. Rev. Ecol. Syst. 6, 339-364

46 Ravigné, V. et al. (2009) Live where you thrive: joint evolution of habitat choice and local adaptation facilitates specialization and promotes diversity. Am. Nat. 174, E141-E169
47 Armsworth, P.R. (2009) Conditional dispersal, clines, and the evolution of dispersiveness. Theor. Ecol. 2, 105-117

48 Armsworth, P.R. and Roughgarden, J.E. (2005) The impact of directed versus random movement on population dynamics and biodiversity patterns. Am. Nat. 165, 449-465

49 Armsworth, P.R. and Roughgarden, J.E. (2008) The structure of clines with fitness-dependent dispersal. Am. Nat. 172, 648-657

50 Davis, J.M. (2008) Patterns of variation in the influence of natal experience on habitat choice. Q. Rev. Biol. 83, 363-380

51 Johnson, C. (1966) Environmental modification of habitat selection in adult damselflies. Ecology 47, 674-676

52 Aubret, F. and Shine, R. (2008) Early experience influences both habitat choice and locomotor performance in tiger snakes. Am. Nat. 171, 524-531

53 Akhtar, Y. and Isman, M.B. (2003) Larval exposure to oviposition deterrents alters subsequent oviposition behavior in generalist, Trichoplusia ni and specialist, Plutella xylostella moths. J. Chem. Ecol. 29, 1853-1870

54 Imbert, E. and Ronce, O. (2011) Phenotypic plasticity for dispersal ability in the seed heteromorphic Crepis sancta (Asteraceae). Oikos 93, 126-134

55 Massot, M. et al. (2002) Condition-dependent dispersal and ontogeny of the dispersal behavior: an experimental approach. J. Anim. Ecol. 71, 253-261

56 Wright, S. (1931) Evolution in Mendelian populations. Genetics 16, 97159

57 Bolnick, D.I. et al. (2008) Migration load in a pair of ecologically divergent lacustrine stickleback populations. Biol. J. Linn. Soc. 94, 373-387

58 Kirkpatrick, M. and Barton, N.H. (1997) Evolution of a species' range. Am. Nat. 150, 1-23

59 Holt, R.D. and Gomulkiewicz, R. (1997) How does immigration influence local adaptation? A reexamination of a familiar paradigm. Am. Nat. 149, 563-572

60 Duckworth, R.A. and Kruuk, L.E.B. (2009) Evolution of genetic integration between dispersal and colonization ability in a bird. Evolution 63, 968-977

61 Duckworth, R.A. and Badyaev, A.V. (2007) Coupling of dispersal and aggression facilitates the rapid range expansion of a passerine bird. Proc. Natl. Acad. Sci. U.S.A. 104, 15017-15022

62 Nosil, P. et al. (2009) Divergent selection and heterogeneous genomic divergence. Mol. Ecol. 18, 375-402

63 Stinchcombe, J.R. and Hoekstra, H.E. (2007) Combining population genomics and quantitative genetics: finding the genes underlying ecologically important traits. Heredity 100, 158-170

64 Hohenlohe, P.A. et al. (2010) Population genomics of paralle adaptation in threespine stickleback using sequenced RAD tags. PLoS Genet. 6, e1000862

65 Coyne, J.A. and Orr, H.A., eds (2004) Speciation, Sinauer Associates

66 Eroukhmanoff, F. et al. (2011) The role of different reproductive barriers during phenotypic divergence of isopod ecotypes. Evolution 65, 2631-2640

67 Dres, M. and Mallet, J. (2002) Host races in plant-feeding insects and their importance in sympatric speciation. Philos. Trans. R. Soc. Lond. B: Biol. Sci. 357, 471-492

68 Holyoak, M. et al. (2008) Trends and missing parts in the study of movement ecology. Proc. Natl. Acad. Sci. U.S.A. 105, 1906019065

69 Lard, M. et al. (2010) Tracking the small with the smallest - using nanotechnology in tracking zooplankton. PLoS ONE 5, e13516 